\title{
Using Electron Microscopy to Enhance the Knowledge of Biological Systems
}

\author{
Ken Ishikawa ${ }^{1}$, Ziqiu Wang ${ }^{2}$, Christina Burks ${ }^{2}$, Mioara Larion ${ }^{3}$, Natalia de Val $^{2 *}$ and Joel Schneider ${ }^{1}$ \\ 1. Peptide Design and Materials Section, Chemical Biology Laboratory, Center for Cancer Research, \\ National Cancer Institute, National Institutes of Health, Frederick, MD, USA. \\ 2. Electron Microscopy Laboratory, Frederick National Laboratory for Cancer Research, Center for \\ Cancer Research, National Cancer Institute, National Institutes of Health, Frederick, MD, USA. \\ ${ }^{3 .}$ Neuro-Oncology Branch, Center for Cancer Research, National Cancer Institute, National Institutes of \\ Health, Bethesda, MD, USA. \\ * Corresponding author: natalia.devalalda@nih.gov
}

The Electron Microscopy Laboratory (EML) supports the Center for Cancer Research (CCR) of the National Cancer Institute (NCI) [1]. The EML offers investigators access to unique expertise and EM technologies that allow our partners to explore new avenues of research in order to study biological systems as well as to help with diagnosis of diseases, mainly cancer related. To assist our collaborators, we provide complete specimen preparation service which includes shipment of fixative, processing, embedding, sectioning, and imaging of samples. If desired, investigators can also discuss the design of custom protocols tailored to their specific needs.

The capabilities that EML offers include Transmission Electron Microscopy (TEM) and Scanning Electron Microscopy (SEM) as well as expertise in the following specialized areas: Immunoelectron microscopy, cryo-electron microscopy, negative stain analysis, ultrastructure analysis and virus particle characterization.

EML provides its expertise in various projects. A current project involves analysis of ultrastructural characteristics of human brain tumor tissue. The morphology of the organelles; golgi, endoplasmic reticulum, mitochondria and lysosomes, were analyzed at high and low magnification for the control and treated samples, to provide accurate and detailed results to the investigator. A quantitative review of the mitochondrial condition provided results for each tumor sample. The mitochondria were categorized under one of the following categories; intact, partial damaged, completely damaged. Lysosomes were measured in all images at 5000x magnification for comparative analysis.

Another project focus on the effects of anti-microbial peptides (AMPs) on eukaryotic cells. AMPs are currently considered as a source of antibiotics and present a potential to become drugs clinically. In the present study, a model AMP SVS-1 was applied on fission yeast cell with a high copy of an alpha-1,2galactosyltransferase gene $(\mathrm{gmh} 2-\mathrm{HC})$ for drug resistance test. EM was used to obtain structural information on cell wall. To guarantee complete infiltration within yeast cell and meanwhile preserve wall structure, a modified infiltration protocol was developed using low viscosity resin. It was found that cell wall structure altered in both electron dense galactomannan and electron lucent glucan layers. Besides, distribution of SVS-1 was visualized via immunoEM by labeling biotin-conjugated SVS-1 with secondary gold particles (Figure 1). In gmh2-HC cells, peptides molecules formed small aggregates, leading to lower local peptide concentration which was insufficient to compromise cell membrane. These results elucidate the association between AMPs and cell wall, and further enhance the understanding of drug resistance behavior in the eukaryotic systems. 


\section{References:}

[1] https://ostr.ccr.cancer.gov/partnerships/imaging/electron-microscopy/

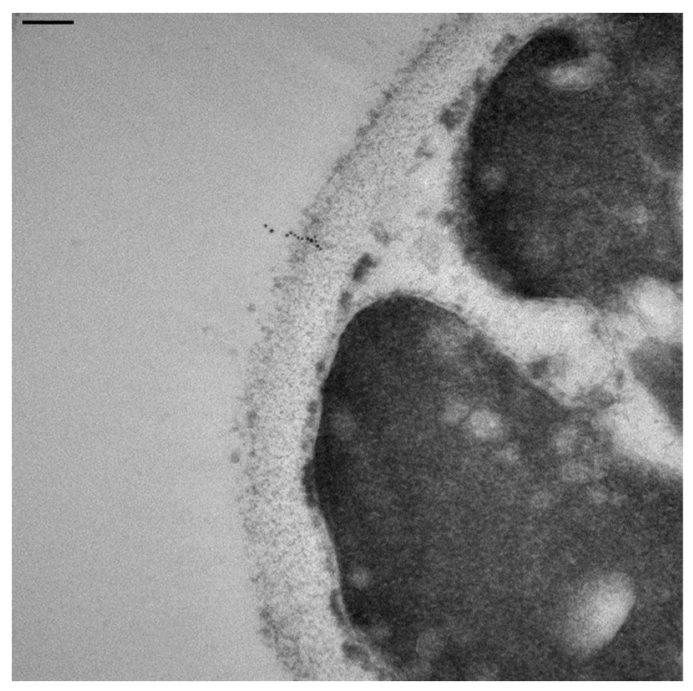

Figure 1. TEM image of yeast cell wall. Biotinylated SVS-1 peptide is visualized by labeling with avidin conjugated gold nanoparticles. Scale bar: $100 \mathrm{~nm}$. 